\title{
Entorno a la definición legal de los recursos naturales y su relación con el derecho constitucional y fundamental a gozar de un ambiente equilibrado y adecuado
}

Environment to the legal definition of natural resources and its relationship with the constitutional and fundamental right to enjoy a balanced and adequate environment

\author{
Juan José Romero Anguerry
}

Universidad Católica de Santa María. Arequipa Perú.

\section{INFORMACIÓN}

\section{Historia del Artículo}

Recepción: 14/05/2019

Revisión: 20/05/2019

Aceptación: 30/08/2019

\section{Palabras Clave}

Recursos naturales, derecho a gozar de un ambiente equilibrado y adecuado, Constitución y medio ambiente.

\section{Key Words}

Natural resources, right to enjoy a balanced and adequate environment, Constitution and environment.

\author{
RESUMEN \\ El estudio jurídico de los recursos naturales debe partir, entre otros, del análisis de la definición \\ legal de los mismos establecida en la Ley $\mathrm{N}^{\circ} 26821$. Dicha definición presenta aciertos y \\ deficiencias, las que se intentan desvelar y analizar. Asimismo, se plantea complementar la \\ conceptualización de los recursos naturales analizando sus nexos con el derecho constitucional \\ y fundamental a gozar de un ambiente equilibrado y adecuado.
}

\begin{abstract}
The legal study of natural resources should start, among others, the analysis of the legal definition of them established in Law No. 26821. This definition presents successes and deficiencies, which are intended to reveal and analyze. Likewise, it is proposed to complement the conceptualization of natural resources by analyzing their links with the constitutional and fundamental right to enjoy a balanced and adequate environment.
\end{abstract}

\section{DOI}

https://doi.org/10.35286/veritas.

v20i2.241

\section{INTRODUCCIÓN}

El tratamiento normativo de los recursos naturales en el Perú es extenso. En cada uno de ellos, se han generado marcos normativos amplios, como ocurre por ejemplo con al régimen de aguas, el minero o el de hidrocarburos. Frente a ello, se considera necesario brindar una visión ordenada del régimen jurídico que abarcan los mismos y, ello necesariamente debe partir de la Constitución. Para abordar el estudio constitucional de los recursos naturales es indispensable hacerlo partiendo de examinar las cuestiones vinculadas al marco conceptual de los mismos y la definición que el Derecho le asigna, lo que como se verá no es un aspecto suficientemente clarificado ni por la dogmática ni por la jurisprudencia del Tribunal Constitucional. A continuación corresponde relacionar el mismo con el derecho constitucional y fundamental al medio ambiente. Estas páginas están dedicadas a dicho propósito formulando como colofón las conclusiones a las que se ha llegado y las recomendaciones correspondientes.
La Constitución de 1993 aborda algunos aspectos centrales del tratamiento jurídico de los recursos naturales como la declaración de patrimonio de la Nación de los mismos y de su uso sostenible y otorgamiento por concesión. Este tratamiento además de tener importantes problemas de ambigüedad resulta ser insuficiente, puesto que no se abordan otros aspectos igualmente importantes y quizá muy necesarios por las circunstancias propias del Perú, como la necesidad de precisar el principio de aprovechamiento sostenible, formular principios para su otorgamiento a terceros, sobre la finalidad de los mismos y en particular del destino de los beneficios que dejan al país producto de su utilización, así como de la forma de superar los conflictos complejos y de distinta naturaleza que se han generado en el país, quizá un aspecto de gran importancia de cara a lograr el desarrollo nacional, apreciando las condiciones actuales. Todo ello requiere de una investigación adecuada y suficiente, de una comprensión de la problemática en función a todas sus aristas y complejidades y de una especial reflexión por todos. Es importante entonces incrementar los estudios sobre este acuciante tema, desde distintos enfoques como el jurídico, en el que está inmerso el presente. 


\section{Análisis en torno a la definición de los recursos naturales}

Muchos de los aspectos centrales con los que el Derecho aborda el régimen general de los recursos naturales se contienen en Ley $\mathrm{N}^{\circ} 26821$, Ley Orgánica de Aprovechamiento Sostenible de los Recursos Naturales (LOASRN), que regula su aprovechamiento sostenible. En la misma se establecen distintas cuestiones como su definición normativa, su referencia patrimonial y alcance, el principio de soberanía, de participación ciudadana, la participación en intervención del Estado y el régimen de otorgamiento de derechos sobre los mismos.

En efecto, el artículo 3 de la LOASRN, se aborda la definición legal de recursos naturales, considerando como tales a todo componente de la naturaleza susceptible de ser aprovechado por el ser humano para la satisfacción de sus necesidades y que tenga un valor actual o potencial en el mercado. Dicho artículo, incluso va más allá y elabora un catálogo de los elementos de la naturaleza que se consideran son recursos naturales (las aguas, los minerales, el suelo, subsuelo, la diversidad biológica, los recursos hidrocarburíferos, hidroenergéticos, eólicos, solares, geotérmicos, entre otros). Tal definición, presenta aciertos, pero también algunos cuestionamientos o críticas, como se pasa a desarrollar.

Esta definición expresa un criterio utilitario de los recursos naturales por los beneficios que de ellos obtenemos centrando los mismos en sus connotaciones económicas, lo que cobra mayor significado, desde que en general, como señala Witker, los recursos naturales se caracterizan por ser limitados e interdependientes. "Limitados, porque no crecen ilimitadamente, puesto que las leyes naturales imponen tasas de crecimiento al grado que cada uno de ellos controla el desarrollo de los demás". Este último referido a que entre ellos se da una relación funcional de equilibrio en que la alteración de uno afecta al desarrollo de los otros ${ }^{1}$. Es en este carácter limitado donde destaca con mayor fuerza la connotación económica, en cuanto que tendrán mayor valor los recursos más escasos y de mayor dificultad para extraerlos o utilizarlos, lo que explicaría la maximización del enfoque económica en la definición legal establecida en la LOASRN.

Gran parte de la dogmática nacional y la jurisprudencia constitucional se han centrado en el tratamiento económico - utilitario de los recursos naturales, del beneficio que los mismos proporcionan al ser humano y, en general a los seres vivos. La utilidad se aprecia desde distintos criterios que se entienden como valores, los que pueden ser sociales, religiosos, culturales y económicos. En este sentido, el Tribunal Constitucional señala que el medio ambiente se compone de los denominados elementos naturales, los cuales pueden generar o no, según sea el caso, algún tipo de utilidad, beneficio o aprovechamiento (material o espiritual) para la existencia o coexistencia humana. Los que generan utilidad son los denominados recursos naturales ${ }^{2}$. Estos, según entiende Andaluz Westreicher, son aquella parte de la naturaleza que tiene alguna utilidad actual o potencial para el hombre, para satisfacer sus necesidades ${ }^{3}$. En esta misma línea, Sánchez Gonzáles, indica que los mismos son aquellos elementos que nos proporciona la naturaleza, de los cuales se sirve el hombre para satisfacer sus necesidades de orden material ${ }^{4}$. El Supremo Contralor de la Constitucionalidad, destaca con mayor fuerza el enfoque utilitario señalando que "los recursos naturales representan aquella parte de la naturaleza que tiene alguna utilidad actual o potencial para el hombre. En otras palabras, son los elementos naturales que el ser humano aprovecha para satisfacer sus necesidades materiales o espirituales; vale decir, que gozan de aptitud para generar algún tipo de provecho y bienestar"s.

Esta orientación hacia la utilidad, ha llevado a autores como Pulgar Vidal a señalar que "la esencia de un recurso natural es su valoración económica, en tanto capacidad o potencial de satisfacer necesidades y, es este grado de valoración el que determina su desarrollo normativo. El nivel de satisfacción de necesidades es el que determina el nivel de valoración"6. Es a partir de esta perspectiva, de aprovechamiento y de generar beneficios económicos al ser humano que ha llevado al Derecho a construir un cuerpo normativo en el que destacan estos. El desarrollo constitucional y legal de los recursos naturales se ha centrado en los aspectos económicos derivados del uso de los mismos, esta es la razón por lo cual uno de los puntos centrales de la formulación normativa se ha sentado en la titularidad sobre ellos. En nuestro país, se asume que los recursos naturales son "patrimonio de la Nación", aspecto que se tratará en otro estudio complementario.

La perspectiva utilitaria de los recursos naturales ha advertido también las circunstancias negativas de la extracción y aprovechamiento desenfrenado de los mismos, por lo que se ha incorporado un principio esencial para su tratamiento jurídico, el de sostenibilidad, que también será abordado igualmente en otro trabajo posterior.

La visión utilitaria económica de los recursos naturales, no solo no es unánime, sino que además ha sido criticada, como lo hace Vignolo Cueva, quien sostiene que "esta perspectiva fue promovida por el Legislador de la liberalización de la década de los noventa. Dicho autor, aprecia más bien que los recursos naturales tienen una funcionalidad múltiple"7. En efecto, la visión económica y utilitaria es parcial y limitada. Ciertamente el referente económico puede ser muy importante, pero no es el único. De hecho, en algunos textos normativos encontramos una perspectiva más amplia. Así, el inciso 1 del Artículo I del Título Preliminar de la Ley de Recursos Hídricos, proclama el principio de valoración del agua, el cual señala que el agua tiene valor sociocultural, valor económico y valor ambiental. En efecto, los aspectos socioculturales, religiosos vinculados a los recursos naturales, adquieren una connotación valorativa de importancia, como también la valoración y connotación ambiental e histórica. Como se aprecia, es necesario considerar a los recursos naturales, no solamente desde su visión económica, sino desde las otras perspectivas igualmente importantes y significativas.

Lo que se critica en el presente estudio no es la referencia utilitaria. El significado usual de recurso es por esencia un elemento útil para atender una necesidad llevar a cabo una actividad ${ }^{8}$; y, es recurso natural, porque proviene de la naturaleza. Lo que cuestiona es su marcado énfasis económico.

Frente a estas cuestiones, Vignolo, formula una propuesta de definición considerando como elementos constitutivos de los recursos naturales los siguientes: la apropiabilidad, en este sentido los mismos tienen indudable cercanía a la visión jurídica de los bienes, pues solo las cosas apropiables permiten su inmediata catalogación jurídica, apropiabilidad que se efectúa respecto a una titularidad específica que 
corresponde a todos los ciudadanos. Los usos se materializan a través de títulos de intervención y dominio público desde las organizaciones administrativas, conforme a ello aprecia que el elemento subjetivo involucra la necesidad de compartir roles entre un dominio público racionalizado y la propiedad privada. Se impone la necesidad de construir una prohibición de una absoluta demanialización estatal de los recursos naturales, pues nunca fueron considerados como cosas apropiables del dominio público, aduce una regla de prohibición de reservas estatales de la que se excluye el suelo, subsuelo, dominio marítimo y espacio aéreo, siendo que en el resto de supuestos el legislador se cuidó de no generar la demanialización produciendo expresiones de una titularidad pública a todos los nacionales. Expresan una funcionalidad múltiple son mezcla entre necesidades de conservación para objetivos de intereses públicos; esta multifuncionalidad, supera el inmovilismo ecologista, entre otros. En relación a la titularidad, señala que se optó por crear una fórmula especial de titularidad pública colectivizada a favor de la Nación que ejerce el señorío y un dominio público singular, siendo que las organizaciones administrativas están para gestionar integralmente los recursos naturales. Se postula que existen dos clases de los mismos: de dominio público colectivo, los grandes elementos de territorio (suelo, subsuelo, dominio marítimo, y espacio aéreo). En relación a la soberanía en su aprovechamiento se debe colocar a las potestades públicas como instrumentos esenciales de la gestión de recursos naturales, siguiendo a Pimiento, sostiene que soberanía es un concepto más político que jurídico que explica las relaciones internacionales e internamente, la soberanía adquiere las características del poder público. Por último, los artículos 54 y 66 de la Constitución Política han creado una figura peculiar de demanio o dominio público natural que intenta excluir del tráfico jurídico privado a todos los recursos naturales a fin de dotarlos siempre de una racionalidad colectiva y de utilización económica9 ${ }^{9}$. Este planteamiento, si bien es más completo que otros, no está exento de crítica, como por ejemplo la posición sobre el dominio público que se desarrollará en otro momento.

Asimismo, respecto a las posibilidades de su utilización, se ha expresado la clasificación más conocida que los distingue entre recursos renovables y no renovables. De hecho, así esta expresada en el artículo 2 de la LOASRN; incluso el Tribunal Constitucional sostiene que los primeros son "aquellos que, pese a ser utilizados, pueden regenerarse; y los no renovables, aquellos que, al ser utilizados, se agotan irremediablemente" ${ }^{10}$. Sin embargo, esta división es cada vez más inexacta e imprecisa. Siguiendo a Figallo, "existe una equivocada creencia que las riquezas son ilimitadas y que pueden renovarse constantemente lo que ha impulsado una desenfrenada e irracional depredación de los recursos". Dicho autor manifiesta que "desde un punto de vista ecológico, en strictu sensu, ningún recurso es totalmente renovable"11. En efecto, el uso irracional y exagerado de ciertos recursos, considerados renovables, está llevando a su escases o posible escases, a lo que se suma las consecuencias presentes y futuras del cambio climático. De manera que la connotación de renovable será puesta cada vez más en cuestión en muchos recursos naturales considerados como tales.

Otro aspecto en torno al concepto de recursos naturales es el de identificar cuando estamos ante la presencia de uno y cuando no. El principio de legalidad es el que marca la pauta de identificación de un recurso natural como tal para el mundo del Derecho. De esta manera, es el legislador el que determina que un elemento de la naturaleza sea considerado como tal. El artículo 3 de la LOASRN contiene, como está dicho, una relación de qué elementos se les considera como recursos naturales, relación que tiene la categoría de "números apertus" al existir la posibilidad de incorporar otros. La definición dada en el citado artículo, en cualquier caso, contribuye a clarificar labores interpretativas, orientando la identificación, pero ciertamente no agotándola, más aún cuando la misma está sujeta a debate.

\section{Los recursos naturales en el marco del derecho constitucional a gozar de un ambiente equilibrado y adecuado}

La formulación constitucional de los recursos naturales, se basa en el contenido del inciso 22 del artículo 2 y en los artículos 66 al 69 de la Constitución, considerándolos elementos esenciales del medio ambiente. A partir de ello, el Tribunal Constitucional, aborda a los recursos naturales tanto como componentes del medio ambiente, como conformantes del derecho constitucional al medio ambiente equilibrado y adecuado para el desarrollo de la persona. Por ello, ha de abordarse previamente el tratamiento constitucional de ambos aspectos.

Tanto la jurisprudencia como la doctrina sientan una relación entre medio ambiente y recursos naturales. El Tribunal Constitucional considera al ambiente "como un sistema; un conjunto de elementos que interactúan entre sí. Implica el compendio de elementos naturales -vivientes o inanimados- sociales y culturales existentes en un lugar y tiempo determinados, que influyen en la vida material y psicológica de los seres humanos. Por dicha razón, es objeto de protección jurídica y forma parte del bagaje de la tutela de los derechos humanos". (...) "El ambiente es concebido como el medio en el cual se encuentran contenidos todos los factores que hacen posible la existencia humana y la de los demás seres vivos". Guillermo Cano, complementando lo dicho aprecia que el ambiente contiene dos categorías, siendo una de ellas: "a) El entorno natural con sus recursos naturales vivos, que comprende la flora, fauna y el sector agrícola y el hombre; y los recursos naturales inertes como las tierras no agrícolas, las aguas, los minerales, la atmósfera y el espacio aéreo, los recursos geotérmicos, la energía primaria y los recursos escénicos o panorámicos"12.

Las implicancias que el medio ambiente tiene para el ser humano han llevado a conformar un derecho fundamental y también constitucional que en el inciso 22 del artículo 2 de la Constitución proclama que toda persona tiene derecho "a gozar de un ambiente equilibrado y adecuado al desarrollo de su vida". En relación a ello, el Tribunal Constitucional ha sostenido que el contenido del derecho fundamental a un medio ambiente equilibrado y adecuado para el desarrollo de la persona está determinado por los siguientes elementos básicos:

1) El derecho a gozar del medio ambiente en su primera manifestación, implica el derecho a gozar de un medio ambiente equilibrado y adecuado, lo que comporta, según el Tribunal Constitucional, "la facultad de las personas de poder disfrutar de un medio ambiente en el que sus elementos se desarrollan e interrelacionan de manera natural y armónica"; la intervención humana no debe alterar sustantivamente esa 
interrelación. Esto implica que solo puede disfrutarse de un entorno adecuado para el desarrollo de la persona y de su dignidad, lo contrario significa vaciar de contenido a dicho derecho $^{13}$.

Este elemento repercute sobre los recursos naturales, pues implica que la intervención humana respecto a los mismos no debe alterar sustantivamente la interrelación antes indicada. Es decir, que el uso y aprovechamiento de tales recursos debe cautelar y conservar las relaciones naturales y armónicas que se presentan en el medio ambiente.

2) El segundo componente lo constituye el derecho a que ese medio ambiente se preserve, esto es el mantenimiento de un medio ambiente sano y equilibrado. Para el Tribunal Constitucional ello implica un mandato directo a todas las personas, primero a todos los poderes públicos, esto es al Estado en su conjunto, en sus tres niveles (nacional, regional y local) y a todas las entidades y órganos que lo conforman, incluidos los de derecho privado, de mantener los bienes ambientales en las condiciones adecuadas para su disfrute; asimismo, a los particulares cuyas actividades económicas inciden, directa o indirectamente en el medio ambiente ${ }^{14}$.

Ambos elementos tienen directas repercusiones o incidencia sobre la obtención, utilización, tratamiento y aprovechamiento de los recursos naturales, constituyendo una limitación y parámetro de actuación para el desarrollo de las citadas actividades, protegiendo en el fondo no solo a la persona humana y su dignidad (valor fundamental del Derecho), sino a la vida en general en el planeta. Considerando lo dicho, se deducen las siguientes implicancias:

El primer elemento repercute sobre los recursos naturales, pues genera una disposición de prevención y protección del ambiente en las condiciones previas a la intervención humana a fin que no se genere una alteración sustantiva en la interrelación de los elementos que conforman el medio ambiente. Esta norma que se deduce del desarrollo expuesto, solo es operativa en circunstancias donde no ha operado la mano del hombre, puesto que lo complicado sería determinar cuáles han sido tales condiciones si la participación antrópica se produjo antes del uso pretendido de los recursos naturales.

El segundo elemento también tiene implicancias sobre el uso de los recursos naturales, pues dirige un mandato urbit et orbi a todas las personas públicas y privadas para que las actividades vinculadas a los recursos naturales se realicen sin alterar las condiciones para el adecuado, suficiente y correcto disfrute del medio ambiente por parte de todos.

Se trata de un mandato obligatorio para los particulares en el desarrollo no solo -aunque sí principalmente- de sus actividades económicas, sino también sociales, culturales, entre otras, lo que trae la consiguiente responsabilidad de asumir las consecuencias de no hacerlo en los distintos órdenes jurídicos (penal, administrativo, civil y ambiental). Esta obligación también y de manera especial está dirigida al Estado en sus actividades, planes o programas de controlar, supervisar, fiscalizar y sancionar las acciones que alteren las interrelaciones que existen entre los elementos naturales.

Asimismo, tiene importancia el desarrollo que se hace del medio ambiente "equilibrado". El Tribunal Constitucional considera que es posible inferir que dentro de su contenido protegido se encuentra el conjunto de bases naturales de la vida y su calidad, lo que comprende, a su vez, sus componentes bióticos, como la flora y la fauna; los componentes abióticos, como el agua, el aire o el subsuelo; los ecosistemas e, incluso, la ecósfera, esto es, la suma de todos los ecosistemas, que son las comunidades de especies que forman una red de interacciones de orden biológico, físico y químico. A todo ello, habría que sumar los elementos sociales y culturales aportantes del grupo humano que lo habite (...) "Nuestra Constitución apunta a que la persona pueda disfrutar de un entorno en simétrica producción, proporción y armonía acondicionada al correcto desarrollo de la existencia y convivencia". El siguiente enunciado "el ambiente debe ser adecuado para el desarrollo de la vida humana", se traduce en la obligación concurrente del Estado y de los particulares de mantener aquellas condiciones naturales del ambiente, a fin de que la vida humana exista en condiciones ambientalmente dignas"15.

Este último aspecto enriquece y precisa los contenidos que se derivan de los dos elementos del derecho al medio ambiente antes desarrollados. En efecto, el Tribunal Constitucional precisa y amplia el ámbito del contenido protegido del medio ambiente al conjunto de bases naturales de la vida y su calidad, comprendiendo dentro de ellos a la totalidad de los recursos naturales.

En base a lo señalado, el Supremo Interprete de la Constitución señala que "la protección comprende el sistema complejo y dinámico de todos sus componentes, en un estado de estabilidad y simetría de sus ecosistemas, que haga posible precisamente el adecuado desarrollo de la vida de los seres humanos" (...) "una interpretación sistemática del artículo 2, inciso 22) y de los artículos 66 y 67 de la Constitución, se concluye que una manifestación concreta del derecho de toda persona a disfrutar de un entorno ambiental idóneo para el desarrollo de su existencia, es el reconocimiento de que los recursos naturales -especialmente los no renovablesen tanto patrimonio de la Nación, deben ser objeto de un aprovechamiento razonable y sostenible, y los beneficios resultantes de tal aprovechamiento deben ser a favor de la colectividad en general, correspondiendo al Estado el deber de promover las políticas adecuadas a tal efecto ${ }^{16}$.

\section{Los recursos naturales como bienes constitucionales}

Como colofón de lo desarrollado, y dada la importancia de los recursos naturales, estos tienen también la consideración de bienes constitucionales; en este sentido, Landa Arroyo señala, que la Constitución ha recogido una serie de bienes calificados como bienes constitucionales reconocidos como valiosos para la convivencia social, cuando no, para la propia existencia del Estado al que le compete un especial deber de protección, entre los cuales se encuentran los recursos naturales, la diversidad biológica, las áreas naturales, entre $\operatorname{otros}^{17}$.

\section{CONCLUSIONES}

La definición del artículo 3 de la LOASRN requiere ser revisada, básica pero no exclusivamente, por su marcado enfoque económico - utilitario que no reconoce otros valores como los sociales, culturales, religiosos y medio ambientales. En este sentido, los recursos naturales tienen, como señaló Vignolo una perspectiva multifuncional. El reconocimiento de otros contextos y valores podría permitir considerar la importancia de otros aspectos y elementos en los múltiples y variados conflictos que respecto a la explotación de los mismos se presenta en el Perú. 
El derecho constitucional y fundamental a gozar de un ambiente equilibrado y adecuado termina de construir y dar sentido a la definición legal de los recursos naturales, generando de dicho principio incluso reglas no solo para la Estado, sino para todas las personas, para toda la Nación. Los intentos de replanteamiento de tal definición no pueden dejar de apreciar esta circunstancia.

\section{REFERENCIAS BIBLIOGRÁFICAS}

1. Witker, Jorge, Curso de Derecho Económico, Universidad Autónoma de México, México, 1989, pp. 71 y 72 .

2. Exp. $\mathrm{N}^{\circ} 0048-2004-\mathrm{PI} / \mathrm{TC}$, fundamento 27.

3. Andaluz Westreicher, Carlos, Manuel de Derecho Ambiental 2da. Edición, Iustitia, LIMA 2009, P. 53.

4. Sánchez González, José, La Protección de los recursos naturales renovables en el derecho mexicano"... Op. Cit, p. 543.

5. Exp. $\mathrm{N}^{\circ} 0048-2004-\mathrm{PI} / \mathrm{TC}$, fundamento 28.

6. Pulgar Vidal Otarola, Manuel, Los Recursos Naturales, el derecho y la visión de desarrollo, en: Revista Ius Et Veritas $\mathrm{N}^{\circ} 36-2008$, Pontificia Universidad Católica del Perú, p. 399.

7. Vignolo Cueva, Orlando, Una definición alternativa sobre los recursos naturales peruanos, en: El Derecho Administrativo como instrumentos al servicio del ciudadano, Palestra, Lima, 2018, pp. 329 y 340.

8. Diccionario de la Real Academia de la Lengua Española, disponible en: www.rae.es, fecha de consulta 14 de enero de 2019.

9. Vignolo Cueva, Orlando, Una definición alternativa sobre los recursos naturales peruanos... op. cit., pp. $333-339$.

10. Exp. $\mathrm{N}^{\circ}$ 2064-2004-AA/TC, Fundamento 5.

11. Figallo A., Guillermo, Régimen de los Recursos Naturales en la Constitución de 1979, en: Derecho PUCP: Revista de la Facultad de Derecho, 1990, p. 314.

12. STC. Exp. $\mathrm{N}^{\circ}$ 0018-2001-AI/TC, fundamento 6.

13. STC. Exp. $\mathrm{N}^{\mathrm{o}}$ 0470-2013-AA/TC, fundamento 13.

14. STC. Exp. $\mathrm{N}^{\circ}$ 1757-2007-PA/TC, fundamento 6.

15. STC. Exp. $\mathrm{N}^{\circ} 0018-2001-\mathrm{AI} / \mathrm{TC}$, fundamento 7.

16. Exp. $\mathrm{N}^{\circ} 0048-2004-\mathrm{PI} / \mathrm{TC}$, fundamento 33.

17. Landa Arroyo, César, Naturaleza jurídica de los recursos forestales y de fauna silvestre. Marco Legaladministrativo y análisis de los vacíos y contingencias legales, en Sociedad Peruana de Ecodesarrollo, Lima, 2014, pp. 11 y 121. 